\title{
Zum chemischen Nachweis postmortaler und intravitaler Lipoidveränderungen im Gehirn
}

\author{
Von F. LINDLAR ${ }^{1}$ ) \\ Aus dem Patbologischen Institut der Freien Universität Berlin (Direktor: Prof. Dr. W. Masshoff)
}

(Eingegangen am 1. Februar 1967)

\begin{abstract}
Das Verhalten der Lipoide bei der Erweichung in der weißen Substanz des Gehirns des Menschen und bei der postmortalen Autolyse wurde untersucht. Aus der Kenntnis der Abbau-Fettsäuren bei der Autolyse und der Ab- und Umbaulipoide bei der anämischen Erweichung lassen sich verwertbare Schlüsse auf passive und aktive Vorgänge ziehen. - Der Abbau der Sphingolipoide erfolgt aktiv und ist an die Gegenwart von Makrophagen gebunden; im Gegensatz dazu sind die Phosphatide auch postmortal und ohne Zelleinwirkung spaltbar. Triglyceride und Cholesterinester entstehen als Umbauprodukte nur intra vitam. Der mit einem Anstieg der Stearin- und Ölsäure einhergehende Schwund der freien und wiederveresterten $C_{20}$ - bis $C_{24}$-Säuren in älteren Nekrosen entspricht einem aktiven intravitalen Prozeß.

The behaviour of lipids in the softening of the white matter of human brain and in post mortem autolysis was investigated. The fatty acids released during autolysis and the lipids from degradation and synthesis during anaemic softening are a useful indication of the passive and active processes taking place. The degradation of sphingolipids is active and depends on the presence of macrophages; on the other hand, the phosphatides are hydrolysed post mortem and without being acted upon by cells. Triglycerides and cholesterol esters only appear as degradation products intra vitam. The disappearance of free and re-esterified $\mathrm{C}_{20^{-}}$to $\mathrm{C}_{24^{-a c i d s}}$, in aged necroses concomitant with the increase of stearic and oleic acids indicates an active intravital process.
\end{abstract}

Unter allgemein-pathologisch interessierenden Aspekten haben wir die chemischen und morphologischen Veränderungen an den Lipoiden zahlreicher Organe während der postmortalen Autolyse untersucht und als hauptsächliche Abbauprodukte mit der Autolysedauer zunehmend langkettige Fettsäuren und - außer im Gehirn - Lysophosphatide gefunden (1-4). Am Beispiel der Hirnsubstanz soll die Abgrenzung intravitaler von postmortalen Vorgängen nach dem Verhalten der Lipoide gezeigt und insbesondere $z u$ der Frage Stellung genommen werden, welche Erscheinungen auf aktive Lebensvorgänge zurückgehen und welche unabhängig von solchen verlaufen.

Für die Untersuchung der postmortalen Veränderungen diente die sterile Autolyse von weißer Substanz des menschlichen Gehirns in vitro. Von dem bei Zimmertemperatur autolysierenden Gewebe wurden nach $1 \mathrm{Std}$. bis zu 24 Tagen Proben entnommen, die Lipoide extrahiert und analysiert (4). Chromatographisch ergibt sich hierbei für die freien Fettsäuren („,FFS“) eine zeitabhängige Zunahme. Die UV-spektrophotometrische Untersuchung der betreffenden aus dem Dünnschichtchromatogramm zurückgewonnenen bzw. säulenchromatographisch abgetrennten FFS ermittelt vor allem Polyensäuren, d. h. $C_{20^{-}}$und $C_{22}$-Säuren mit 4,5 und 6 Doppelbindungen. Diese Säuren können nur aus den Glycerinphosphatiden der Hirnlipoide stammen (5). Erste Untersuchungen der FFS mit der Gaschromatographie lassen für das Auftreten von freien $\mathrm{C}_{24}$-Säuren, die im normalen Gehirn säureamidartig gebunden nur in den Sphingolipoiden vorkommen, keinen sicheren Anhalt gewinnen. Der Phosphorgehalt der Gesamtlipoidextrakte nimmt nach 24-tägiger Autolyse um etwa die Hälfte ab; die Sphingolipoide bleiben chromatographisch unverändert.

Aus diesen Ergebnissen wird geschlossen, daß bei der postmortalen Autolyse der weißen Hirnsubstanz offen-

1) Nach einem am 5.12.1966 an der Ruhr-Universität Bochum gehaltenen Vortrag. Die Untersuchungen wurden durch die Deutsche Forschungsgemeinschaft unterstützt. sichtlich nur Phosphatide abgebaut werden. Dieser Lipoidabbau ist als überwiegend passiv anzusehen, da er auf jeden Fall ohne Mitwirkung von Zellen und zumindest nach etwa 12 Tagen nach histochemischen Befunden anenzymatisch erfolgt.

Für das Studium entsprechender intravitaler Veränderungen mit und ohne zellige Reaktion wurden anämische Erweichungsherde des menschlichen Gehirns herangezogen (6). Bekanntlich wird den nach etwa 2 Tagen in der Erweichung auftretenden Fettkörnchenzellen eine besondere Rolle beim Abbau (und Abtransport) der Myelinlipoide zugesprochen, ohne daß die Abbauvorgänge bis in ihre Einzelheiten bekannt sind. Vorerst gilt daher das von Rossiter (7) für die durchtrennten peripheren Nerven der Katze entwickelte Schema auch für die anämische Nekrose der weißen Hirnsubstanz. Nach diesem Schema werden aus Sphingolipoiden und Phosphatiden der Nervensubstanz neben hydrophilen Abbauprodukten Fettsäuren frei, die mit Glycerin sich zu Triglyceriden und mit dem primär freien Cholesterin zu Cholesterinestern verbinden. JATZKEwITZ (8) und SCHMIDT (9) konnten das Rossiter-Schema bereits dahingehend erweitern, daß die aus der Säureamidbindung in den Sphingolipoiden abgespaltenen $\mathrm{C}_{24}$-Säuren $z$. T. eine Kettenverkürzung erfahren, daneben aber auch in ungekürzter Form mit Glycerin und Cholesterin verestert werden können.

Neben Fragen, die den Myelinabbau durch Makrophagen betreffen, war zu prüfen, was mit den Myelinlipoiden vor dem Auftreten der Phagocyten, also azellulär geschieht $(4,6)$. Nach den Befunden an der autolysierenden weißen Hirnsubstanz war damit zu rechnen, daß zumindest Phosphatide in ganz frischen anämischen Nekrosen auch ohne Beteiligung von Makrophagen gespalten werden können.

Bei ersten orientierenden Untersuchungen zeigte sich, $\mathrm{da} a$ sich der Modus des Lipoidabbaues mit der papierund dünnschichtchromatographischen Analyse der Phosphatide und Sphingolipoide nicbt aufdecken ließ. Dies dürfte im wesentlichen in der heterogenen $\mathrm{Zu}$ - 
sammensetzung der Lipoide in der anämischen Nekrose begründet sein, in der sich präexistente mit $\mathrm{Ab}$ - und Umbausubstanzen in unterschiedlichem Verhältnis durchmischen. Erst die Anwendung der Dünnschichtchromatographie mit der Möglichkeit zur Trennung von Glyceriden, freien Fettsäuren, Cholesterin und seinen Estern (10) bot einen Ansatz, im Anschluß an die Identifizierung von $\mathrm{Ab}$ - und Umbaulipoiden diese aus dem Chromatogramm zurückzugewinnen und die so isolierten Substanzen näher zu charakterisieren (4). Neben Fettsäuren als Abbaulipoiden, die zusätzlich zu den bei der Autolyse nachgewiesenen auftreten, handelt es sich in erster Linie um Triglyceride und Cholesterinester; die beiden letzten bezeichnet man als „Umbaulipoide“. Diese drei Substanzgruppen lassen sich in allen untersuchten Herden verschiedenen Alters (ein Tag bis mehrere Monate) in recht unterschiedlicher Menge auffinden.

Die gaschromatographische und UV-spektrophotometrische Analyse der FFS und der (sekundär) glyceridund cholesterinestergebundenen Fettsäuren ergab einige bemerkenswerte Befunde, deren wichtigster die Polyensäuren und die Lignocerinsäure betriftt (6). Polyensäuren treten in freier und wiederveresterter Form bereits in einem frischen von Fettkörnchenzellen freien Herd auf. Lignocerinsäure erscheint erst, wenn Makrophagen gebildet sind. In einer 5 Wochen alten Nekrose sind Polyensäuren stark vermindert, Lignocerinsäure ist so gut wie vollständig geschwunden. $\mathrm{Da}$ wie bereits dargelegt im Gehirn Polyensäuren nur aus Glycerinphosphatiden stammen, Lignocerinsäure dagegen nahezu ausschließlich aus Sphingolipoiden, muß geschlossen werden, daß die letztgenannten nur in Gegenwart von Makrophagen, Phosphatide aber wie erwartet auch bei Abwesenheit derselben gespalten werden können. Die starke Abnahme bzw. der Schwund der betreffenden Säuren in der älteren Nekrose kann nur durch eine Kettenverkürzung bzw. einen vollständigen Abbau erklärt werden. Für die Kettenverkürzung spricht die Tatsache, daß sich innerhalb der Ab- und Umbaufettsäuren synchron zur Abnahme der Polyensäuren und der Lignocerinsäure ein Anstieg der Stearin- und Ölsäure nachweisen läßt. Ob zusätzlich zur Kettenverkürzung der $\mathrm{C}_{20^{-}}$bis $\mathrm{C}_{24^{4}}$-Säuren eine Hydrierung der hochungesättigten Komponenten erfolgt, hat sich noch nicht klären lassen.

\title{
Literatur
}

1. Lindear, F., Naturwissenschaften 49, 543 (1962). - 2. MassHOFF, W., F. LindlaR und H. J. STOlpManN, Virchows Arch. path. Anat. 337, 340 (1964). - 3. LindLAR, F. und I. A. ZAKI, Virchows Arch. path. Anat. 341, 142 (1966). - 4. LindlaR, F. und R. GürTLER, Acta neuropath. (Berlin) 6, 349 (1966). - 5. KLENK, E. und F. Lindlar, Hoppe-Seyler's Z. physiol. Chem. 299, 74 (1955). -
6. Lindlar, F. und F. Lorenz, Acta neuropath. (Berlin), im Druck. - 7. Rossiter, R. J. in K. A. C. Elliot, I. H. Page und J. H. QuAstex, Neurochemistry. Thomas, Springfield, Ill. (1955). 8. Jatzkéwrtz, H. und E. Merr, Hoppe-Seyler's Z. physiol. Chem. 329, 264 (1962). - 9. Schmidt, J., Diss. München (1965). 10. Mangold, H. K. und N. Tuna, Federat. Proc. 20, 268 (1961).

Priv.-Doz. Dr. F. Lindlar

1 Berlin 19, Spandauer Damm 130

\section{Zur Bestimmung von Ketosteroiden mit 2,4-Dinitrophenylhydrazin}

\author{
II. Mitteilung: Anvendung der Methode bei Plasmaanalysen ${ }^{1}$ )
}

Von L. Treiber, W. Rindt und G. W. Oertel

Aus der Abteilung für Experimentelle Endokrinologie derUniversitäts-Frauenklinik Mainz (Direktor: Prof. Dr.v.Friedberg)

(Eingegangen am 20. Juni 1966)

\begin{abstract}
Dehydroepiandrosteron, Androsteron und Ätiocholanolon, freies Ätiocholanolon und Progesteron können in Plasmaextrakten mittels der 2,4-Dinitrophenylhydrazin-Reaktion als Endpunktsbestimmung quantitativ nachgewiesen werden. Eine Dünnschichtchromatographie der freien, bzw. der aus Konjugaten freigesetzten Steroide, sowie der entsprechenden 2,4Dinitrophenylhydrazone sorgt für die Spezifität der einzelnen Verfahren, die bei einer Richtigkeit um $80 \%$ und einer Genauigkeit von rund $6-10 \%$ die Erfassung von $0,1-0,2 \mu \mathrm{g}$ Steroid gestatten. Die Ergebnisse, die im Verlauf der Analyse von 5-10 $\mathrm{ml}$ Plasma erzielt wurden, entsprechen denjenigen anderer, be: währter Methoden.

Dehydroepiandrosterone, androsterone and etiocholanolone, free etiocholanolon and progesterone can be assayed in plasma extract by means of the 2,4-dinitrophenylhydrazine reaction as an end point determination. Thin layer chromatography of the free steroids, those liberated from conjugates, and the 2,4-dinitrophenylhydrazones ensures the specificity of the individual methods, which at an accuracy of aproximately $80 \%$ and a precision of $6-10 \%$ allows the estimation of $0,1-0,2 \mu \mathrm{g}$ steroid. Results obtained by the analysis of $5-10$ $\mathrm{m} l$ plasma agree with those obtained from other qualified procedures.
\end{abstract}

Wie aus der vorangegangenen Mitteilung (1) zu erkennen ist, läßt sich die Reaktion von Ketosteroiden mit 2,4-Dinitrophenylhydrazin für die quantitative Bestimmung derartiger Verbindungen erfolgreich benutzen. Die empfindliche Farbreaktion, die bei Ver-

1) Vorliegende Arbeit wurde mit Unterstützung der Deutschen Forschungsgemeinschaft, Bad Godesberg, durchgeführt. wendưng von Mikroküvetten den Nachweis von beinahe $0,1 \mu \mathrm{g}$ Mono-ketosteroid gestattet, sollte daher die Analyse solcher Steroide erleichtern, die im Untersuchungsmaterial nur in niedrigen Konzentrationen vorhanden sind, oder aber die Erfassung gesuchter Ketosteroide in geringen Volumina des jeweiligen Ausgangsmaterials erleichtern. Nachstehende Untersuchungen be- 